\title{
Réséda Ponga et Laurence Lagabrielle, Conte kanak
} en français-a’jië

Éd. Grain de sable jeunesse et Centre culturel Tjibaou, Nouméa, 2005

Isabelle Leblic

\section{(2) OpenEdition}

\section{Journals}

Édition électronique

URL : http://journals.openedition.org/jso/497

DOI : $10.4000 /$ jso.497

ISSN : $1760-7256$

\section{Éditeur}

Société des océanistes

Édition imprimée

Date de publication : 1 décembre 2005

Pagination : 201-202

ISSN : 0300-953x

Référence électronique

Isabelle Leblic, "Réséda Ponga et Laurence Lagabrielle, Conte kanak en français-a'jië », Journal de la Société des Océanistes [En ligne], 120-121 | Année 2005, mis en ligne le 01 septembre 2008, consulté le 24 septembre 2020. URL : http://journals.openedition.org/jso/497 ; DOI : https://doi.org/10.4000/jso. 497 
sement en 1958, ou que l'arrivée de la LMS $^{3}$ en 1797 (p. 176).

En conséquence, la description de la crise fait la part belle aux stratégies politiciennes et à la psychologie des deux protagonistes, toutes choses que Regnault analyse avec finesse, mais qui régaleront surtout l'accro des médias qui "affriole" anecdotes pittoresques ou one-man show dramatique. Au bout du compte, c'est forcément au sociologue et au militant que doit revenir le sujet extraordinairement difficile du changement en profondeur, et non au spécialiste de science politique. Attendons de voir ce qu'ils en feront après quarante années galopantes de modernisation exogène !

L'auteur dédie son travail aux humbles, aux petits, à ceux que l'on n'entend jamais (p. 3). Il voudrait qu'ils puissent avoir enfin la parole. Qui ne s'associerait à son vœu? Et, de fait, l'image sempiternelle de Tahiti comme Paradis, comme île bienheureuse où n'existeraient ni conflits ni désespoir et qui fait rêver les touristes, est une invention des hommes d'influence, véhiculée par les hommes d'influence. On n'a jamais demandé aux Tahitiens les plus humbles s'ils s'y reconnaissaient. D'ailleurs, même Oscar Temaru ne voit probablement pas les choses comme eux. Malgré les valeurs qu'il incarne et le courage qui l'anime, il fait partie des privilégiés avec des revenus mensuels en 2001 dépassant 200000 de nos francs (p. 76).

$\mathrm{Si}$ bien que le duel Flosse/Temaru et cette crise spectaculaire qui ont passionné les médias risquent d'apparaître un jour prochain comme un théâtre d'ombres sans rapport avec les problèmes dans lesquels se débat la masse de la population, notamment celle qui vit ou survit sur le "côté montagne".

Comme le citoyen de métropole a aussi son mot à dire, à l'égal de $\mathrm{M}^{\mathrm{me}}$ Girardin, il osera considérer la folle prodigalité du bailleur de fonds et le gaspillage cynique du bénéficiaire et il en viendra peut-être à souhaiter la rupture du lien pervers qui unit la France à ce territoire "autonome". Du coup, il rêvera que la victoire de Temaru annonce le commencement de la fin, ou au moins un peu de démocratie et un autre partage des richesses?

Ce livre connaîtra certainement une nouvelle édition $^{4}$, il la mérite. Mais il faudra que l'auteur le remanie en un ensemble plus cohérent. Aujourd'hui, il ressemble à un tifaifai $^{5}$ bigarré, avec des morceaux empruntés à ses publications antérieures et d'autres fraîchement rédigés dans le feu de l'actualité. On comprend qu'il se soit hâté de produire son travail. Mais le calme revenu, nul doute qu'il prendra le temps de coordonner les éléments disparates, de soigner la mise en page, de reformater la bibliographie qui est peu lisible et enfin de créer un index des noms propres nous guidant vers les faits et dits des Vernaudon, Léontieff et autres Didier Julia (le fameux spécialiste chiraquien des problèmes exotiques !)

\section{Michel PANOFF}

Réséda Ponga et Laurence LAGABRIELle, 2005. Mèyènô. Conte kanak en français-a’jië, Nouméa, Éd. Grain de sable jeunesse et Centre culturel Tjibaou, 28 p., illustrations, lexique, $\mathrm{CD}$ audio bilingue français-a'jië.

Cet ouvrage est le deuxième album d'une série de contes kanak contemporains, illustrant l'émergence d'une littérature destinée à la jeunesse calédonienne, initiée par l'Agence de développement de la culture kanak et les éditions Grain de sable jeunesse. En associant un texte bilingue, ajië-français, cet album permet au lecteur de se familiariser avec le patrimoine culturel et linguistique kanak, à travers une création contemporaine ${ }^{6}$. Dans ce cadre, la présence dans cet ouvrage d'un CD également bilingue est une bonne initiative qui permet au jeune lecteur de se familiariser avec l'une ou l'autre des langues. Mais revenons rapidement à l'histoire.

Méyènô est un garçon kanak très curieux, en quête de savoir comme nombre de garçons de son âge, et qui vit avec son grand-père Mörua aux pays des ruisseaux. Méyènô signifie d'ailleurs en ajië celui qui « recherche la parole » alors que Mörua est celui qui a vécu longtemps. Comme tous les enfants, il ne cesse de poser des questions à son grand-père sur le monde qui l'entoure. Mais les réponses de son grand-père ne lui suffisent pas et il décide un jour de partir à la découverte de ce monde et de suivre la rivière jusqu'à la mer... pour savoir où elle va! Et le chemin suivi a tout d'un itinéraire kanak.

En effet, si cette quête peut paraître universelle, elle est également très kanak et les thèmes abordés ne sont pas sans rappeler ceux que l'on trouve dans la tradition orale: de l'eau source de vie, à la mer domaine du pays des morts, en passant par le lézard animal totémique, l'importance des ancêtres et des esprits des morts qui supportent les vivants et leurs actions... En arrivant au bord de mer, Méyènô est attiré par les profondeurs. Le lézard prévient le grandpère qui part pour retrouver son petit-fils au pays des morts. Cette quête est donc aussi un voyage au pays sous-marin des morts ! Et le grand-père et son petit-fils se retrouvent pour l'éternité au pays des morts avec tous leurs ancêtres.

Cette histoire est très joliment accompagnée d'illustrations aux tons chauds, rendant à merveille l'atmosphère des paysages calédoniens avec des personnages en silhouette, sans traits visibles, permettant à tout

3. NDLR. London Missionary Society.

4. NDLR. Ce livre est aujourd'hui épuisé dans son édition tahitienne. Mais il a été réédité en 2005 en métropole sous le titre Le pouvoir confisqué en Polynésie française. L'affrontement TemarulFlosse, Paris, Les Indes savantes, coll. Asie.

5. NDLR. Couverture polynésienne en patchwork.

6. Le premier ouvrage, Téâ Kanaké, était bilingue paicî-français et reprenait un grand classique de la tradition orale paicî. 
jeune lecteur de s'identifier au personnage, dans un univers constitué des éléments de la nature et de la surnature kanak.

Moyen tout à la fois de valoriser le patrimoine kanak et la créativité artistique actuelle, la publication de ce type d'ouvrage est destinée tout d'abord à la jeunesse calédonienne. Ce genre d'initiative est à encourager car elle permet d'apporter des ouvrages de qualité pouvant servir également à l'enseignement des langues kanak dans les écoles. En cela, le petit lexique bilingue pour expliquer les mots et leurs significations symboliques est des plus utile.

L'auteur et l'illustratrice, Réséda Ponga (originaire de Kouaoua) et Laurence Lagabrielle, ont toutes deux bénéficié, chacune dans leur domaine - l'écriture pour la première et l'illustration pour la seconde - d'une formation au centre culturel Tjibaou. Espérons que d'autres suivront cette voie et nous offriront bientôt d'autres ouvrages de cette qualité.

Isabelle LEBLIC CNRS - LACITO

Christine A. Hemming, 2000. The Art of the French Voyages to New Zealand. 1769-1846, Auckland, Heritage Press Ltd. 91 p.

Ce petit ouvrage est le résultat d'une recherche effectuée en 1998-1999 par Christine Hemming dans le cadre d'une bourse du ministère des Affaires étrangères français. Le texte d'une douzaine de pages (pp. 1324) est bref, mais le regroupement de reproductions de documents rares et peu connus fait l'intérêt de ce livre. En préambule, trois pages (pp. 9-11) sont consacrées à célébrer l'aventure de la diffusion à travers le monde, depuis 1772, d'une des plus grandes marques françaises de champagne qui a sponsorisé cette publication. Cette grande aventure a abouti, en 1985, à un partenariat avec une société viticole néo-zélandaise dont les vignobles s'étendent dans la région de Marlborough, à l'ouest de la vallée de Wairau que le capitaine Cook visita en 1770 .

Dans la première partie de son texte d'accompagnement (pp. 13-18), Christine Hemming montre comment les expéditions françaises de la fin du XVIII ${ }^{\mathrm{e}}$ siècle et de la première moitié du XIX ${ }^{\mathrm{e}}$ siècle se situent dans le sillage des idées humanistes qui émergèrent à l'époque de la Révolution française. Elle expose également comment la création, par les gouvernements postrévolutionnaires, de sociétés savantes, comme la Société des Observateurs de l'Homme, la Société d'histoire naturelle et l'Académie des Sciences, encouragea la publication des résultats des recherches accomplies durant ces voyages dans l'océan Pacifique. Pour l'auteur, les illustrations rapportées viennent confirmer que les objectifs de ces expéditions françaises furent peut-être moins pragmatiques et utilitaristes que celles effectuées par les Britanniques. Elles permirent à la France de développer une recherche scientifique de haut niveau. Ainsi, les spécimens rapportés de Nouvelle-Zélande furent examinés par les plus grandes personnalités scientifiques du nouvellement créé
Museum national d'histoire naturelle: Georges Cuvier, Isidore Geoffroy Saint-Hilaire, Adolphe Brongniart et Bory de Saint-Vincent. Les illustrations réalisées par les artistes embarqués à bord des navires sont les reflets de la pensée d'une époque par le choix des sujets et de leurs interprétations. Christine Hemming s'est attachée à rechercher, dans leurs lieux de conservation, les originaux de ces illustrations qui ont été maintes fois copiées et réinterprétées selon l'imagination des copistes et des graveurs. Cependant, il faut reconnaître que leurs publications en a permis une grande diffusion à travers l'Europe et le Nouveau-Monde. À une époque où la photographie n'existait pas encore, ces images demeurent les seuls témoignages visuels de la vie de communautés, comme celle des Maori, dont l'existence a été depuis profondément modifiée par la colonisation européenne.

Dans une seconde partie (pp. 18-24), l'auteur présente la dizaine d'expéditions françaises qui firent escales en Nouvelle-Zélande de 1769 à 1846, après le voyage de Louis-Antoine de Bougainville qui ne visita pas ce pays. Surville fut le premier Français à longer les côte de l'île nord de la Nouvelle-Zélande en 1769, la même année que le capitaine Cook. La peinture de Pottier de l'Horme représentant le chef maori Ranginui (p. 29), capturé par les marins français avant leur départ, sera reprise dans de nombreuses publications pendant plusieurs dizaines d'années. Les dessins d'armes et d'instruments maori divers, comme ceux de villages fortifiés, sont parmi les premiers connus. La seconde expédition française en Nouvelle-Zélande, en 1772, se termina par l'assassinat de son commandant Marion du Fresne par les Maoris. Elle rapportera cependant de beaux dessins de fortifications et d'embarcations (pp. 32-34). Vingt ans plus tard, en 1793, le capitaine Bruny d'Entrecasteaux, parti à la recherche des deux bateaux disparus de Lapérouse, l'Astrolabe et la Boussole, découvrit la côte nord du pays. Durant cette visite, le peintre Piron réalisera de beaux dessins de Maoris et des croquis de poissons locaux (pp. 37-38).

Il faudra attendre le $\mathrm{XIX}^{\mathrm{e}}$ siècle avec le voyage de Duperrey, en1824, pour voir de nouveaux Français en Nouvelle-Zélande. La réussite de cette expédition scientifique, particulièrement fructueuse, est en partie due à la présence du jeune botaniste René-Primevère Lesson, attaché à l'École navale de Rochefort, et du peintre Jules-Louis Lejeune qui réalisèrent une grande quantité de dessins, dont certains furent réinterprétés, parfois curieusement, à Paris par Antoine Chazal (pp. 39-47). Le plus populaire des explorateurs de cette région est Sébastien-César Dumont d'Urville qui effectua deux séjours en Nouvelle-Zélande, en 1827 et en 1840. L'artiste officiel de sa première expédition, Louis-Auguste Sainson, produisit de très belles aquarelles (pp. 50-52). Edmond-François Pâris dessina de nombreux croquis de bateaux indigènes (p. 62) qui serviront à la réalisation de maquettes conservées aujourd'hui au musée de la Marine. De nombreuses publications scientifiques, illustrant la vie zoologique et botanique néo-zélandaises, furent réalisées à partir de dessins rapportés par Jean-René Constant Quoy et 